\title{
Cellulose production and the evolution of the sessile lifestyle in ascidians
}

\author{
Yasunori Sasakura* \\ Shimoda Marine Research Center, University of Tsukuba, Shimoda, Shizuoka 415-0025, Japan \\ * Corresponding author: Yasunori Sasakura E-mail: sasakura@shimoda.tsukuba.ac.jp \\ (Received April 24, 2018; Accepted June 1, 2018; Published Online July 31, 2018)
}

\begin{abstract}
The outstanding characteristics of the marine invertebrate chordates, ascidians, are a sessile adult stage and cellulose production. These characteristics are not seen in other chordate groups. Molecular studies have suggested that these two characteristics are tightly linked. Ascidians possess the gene encoding cellulose synthase in their genomes. The disruption of the cellulose synthase gene results in abnormal metamorphosis and failure in adhesion, suggesting that cellulose is necessary for starting and continuing sessile life. Ascidian cellulose synthase is suspected to have been transferred to the tunicate ancestor from a bacterial group by horizontal gene transfer. It was suggested that by this transfer, the ancestor of tunicates was given both the gene body and an epidermal enhancer from the bacterium. The simultaneous transfer of these two genetic elements is thought to have facilitated the success of the gene transfer, which enabled the ancestor to utilize cellulose for evolving sessile ascidians.
\end{abstract}

Keywords: tunicate, ascidian, Ciona, cellulose, metamorphosis, horizontal gene transfer

\section{Introduction}

The phylum (or superphylum) Chordata (Fig. 1a) consists of three groups: cephalochordates, tunicates, and vertebrates (Satoh et al., 2014). Among these groups, the cephalochordate group is at the most basal position according to the phylogenetic analyses, and the tunicate and vertebrate groups form sister groups with each other (Delsuc et al., 2006). Animals classified into the phylum chordate share some outstanding characteristics that can be used to distinguish them from other animal groups. For example, chordates have a notochord at a certain period of their life and a tubular central nervous system (CNS) at the dorsal part of the body. Most chordates are free-living animals; they have developed muscle and are motile at both their larval and adult stages. These characteristics enable us to recognize a chordate species relatively easily, except for ascidians whose adults are difficult to be viewed as chordates or even as animals.

Tunicates include thaliaceans and larvaceans in addition to ascidians, and ascidians form the largest group among the tunicates. Adult ascidians generally have a vase-like shape (Fig. 1b), and they are immotile, sessile animals. The sessile lifestyle is the characteristic specific to ascidians among the tunicates. Other tunicate groups such as larvaceans and thaliaceans are planktonic throughout their life. The CNS of ascidian adults are small and short compared to their entire body size, and the dorsoventral axis of the CNS is not easy to recognize from its outside morphology. This is in contrast to the CNSs of other chordates; for example, the vertebrate CNS has a long spinal cord. A notochord is not found in adult ascidians. The overall morphology of adult ascidians is quite distinct from that of the typical tadpole structure, which is the representative shape of chordates, and ascidians were previously misclassified with a different animal group (Satoh, 2003).

The chordate features of ascidians can be easily seen in their larval body. The ascidian larva, unlike ascidian adult, exhibits the typical tadpole shape (Fig. 1c). The larva has a rodlike body with a thicker trunk and a long and thinner tail. The tail includes a notochord at the center, surrounded by muscles that have striated muscle fibers. The larval CNS is located at the dorsal side. The CNS is formed by closure of the neural tube, which is a characteristic morphogenetic movement that is also seen in other chordates for the formation of a tubular CNS (Nicol and Meinertzhagen, 1988; Ogura et al., 2011). The thickened anterior side of the ascidian larval CNS is due to the gathering of neurons to form the sensory vesicle that is comparable to the brain of vertebrates (Wada et al., 1998; Dufour et al., 2006; Ikuta and Saiga, 2007). The CNS of the ascidian larva includes motor neurons that regulate the contractility of tail muscles for swimming (Horie et al., 2010; Stolfi and Levine, 2011; Nishino et al., 2011; Ryan et al., 2016). By using the motor system, ascidians have a motile lifestyle at the larval stage. The shape of ascidian larvae is distinct from that of adults, and the distinctness has made it difficult to infer their relationships.

The shape difference between larval and adult ascidians is achieved by metamorphosis (Cloney, 1982). The ascidian larva possesses an adhesive organ named adhesive papilla at 
(a)
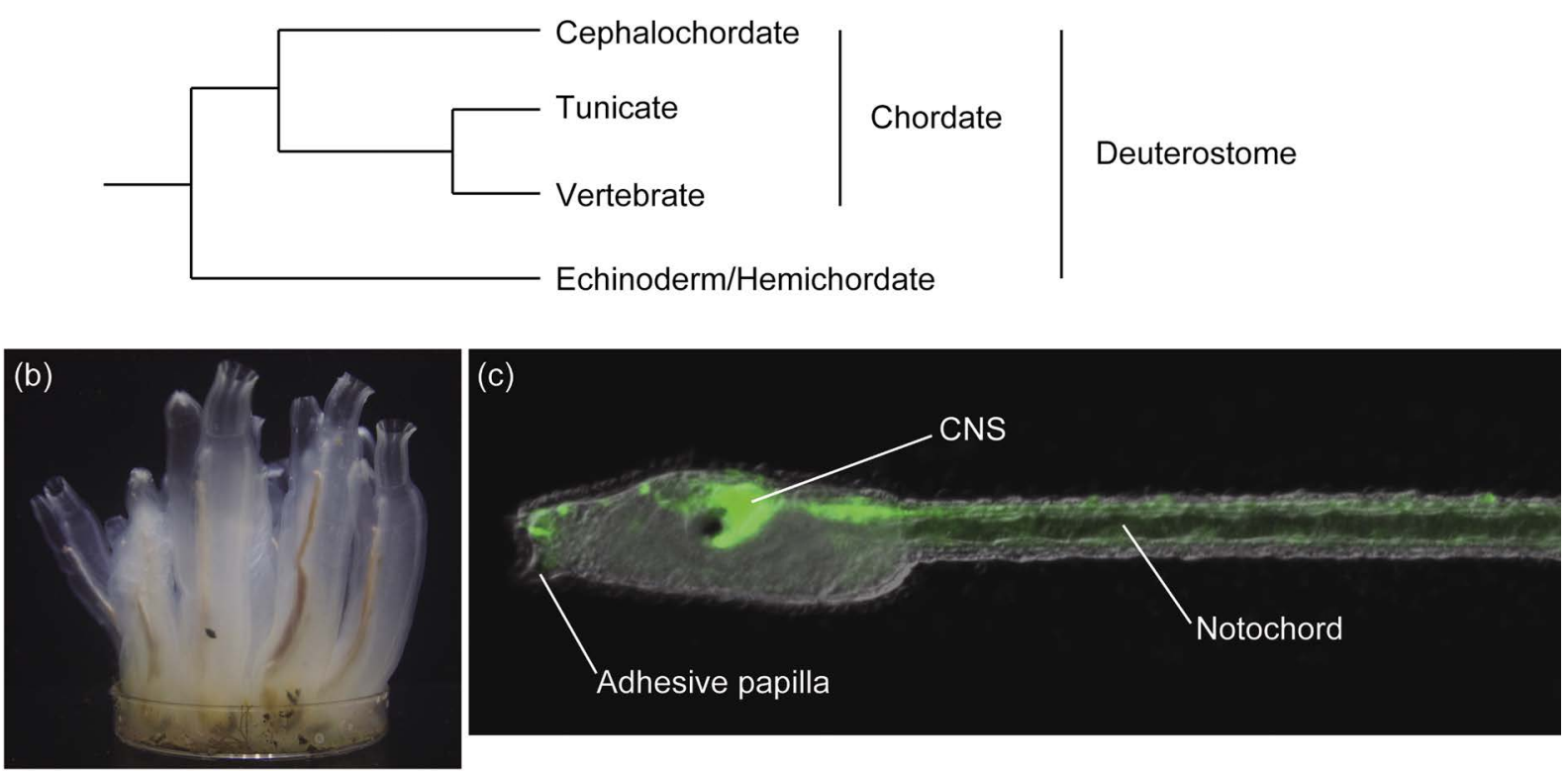

Fig. 1. Chordates, tunicates and ascidians. a: Phylogenetic position of tunicates in relation to the other chordate groups. b: Adults of the ascidian Ciona intestinalis Type A. c: A larva of Ciona intestinalis Type A. Green represents the position of the nervous systems. CNS, central nervous system.
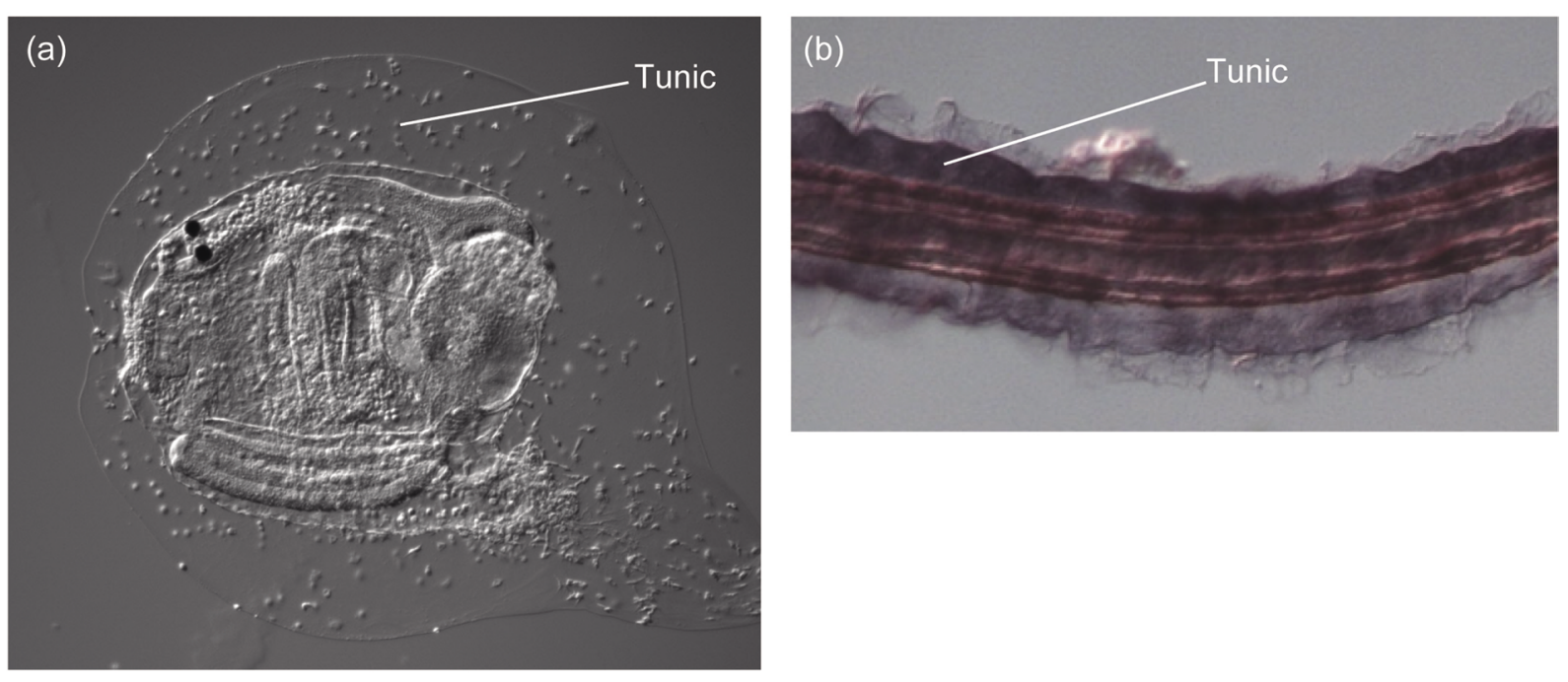

(c)

Ci-CesA (1277 aa)

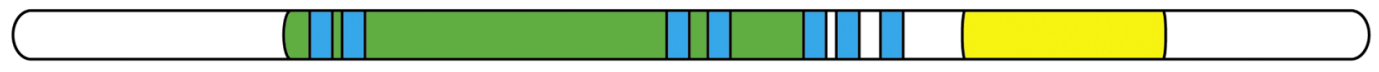

\section{Transmembrane domain \\ Region similar to \\ cellulose synthase}

Region similar to

GH-6 cellulase

Fig. 2. The tunic of ascidians contains cellulose. a: A juvenile of $C$. intestinalis Type A. The animal is covered with the transparent tunic. This animal is approx. 1 week after fertilization, and the larval tail was lost by the metamorphic event. b: Cellulose staining of the larval tunic. The brown color represents the presence of cellulose. c: Schematic illustration of Ciona CesA protein. Left=the N-terminal of this protein. aa, amino acid. 
the anterior pole of the trunk (Fig. 1c). The adhesive papilla secretes viscous substances, and the larva adheres to the substrate with the papilla. The papilla is a neuronal organ (Horie et al., 2008), and adhesion is thought to be the stimulus that starts the excitation of the neurons in the papilla. The excitation of the neurons could be transmitted toward the posterior part of the body through neurites (Takamura, 1998), and a set of metamorphic events is initiated.

The outstanding metamorphic event of ascidians is the loss of the tail. Most body parts of the adult ascidian are formed by the cells at the larval trunk supplemented by the ventrally located tail cells consisting of the endodermal strand and primordial germ cells (Hirano and Nishida, 1997, 2000; ShiraeKurabayashi et al., 2006; Nakazawa et al., 2013; Kawai et al., 2015). Other cells that form the larval tail are lost during metamorphosis by tail regression and apoptosis (Chambon et al., 2002, 2007). By this metamorphic alteration, ascidians lose their apparent chordate features and motility to start sessile life.

For sessile organisms, protection of their body is essential for defense against predators. Ascidians achieve this defense by the tunic covering of their fragile body (Fig. 2a). The tunic is the outmost layer of ascidians; it is not hard compared to the shells of mollusks, but the tunic is so flexible that it is not easily torn. The ascidian tunic contains cellulose (Fig. 2b; Ranby, 1952), the organic macromolecule that is well known as the major component of cell walls of plant cells. Cellulose provides toughness to the ascidian tunic (Sasakura et al., 2005). The production of cellulose is not a specific feature of ascidians, and the ability to produce cellulose is shared among tunicate groups (Hirose et al., 1999). However, among the chordates, the tunicates are the only group that synthesizes cellulose. This situation is not restricted to chordates; the ability to produce cellulose is an exceptional characteristic of tunicates among all animal groups. To understand the sessile lifestyle of ascidians, it is thus necessary to address the mechanisms underlying the production of cellulose by tunicates.

\section{Ascidians produce cellulose for themselves}

The draft genome of the ascidian Ciona intestinalis (currently there is controversy regarding the species name of this ascidian; however, I use this name according to the original manuscripts that I refer to herein) was reported by Dehal et al. in 2002. Extensive investigations and annotations of genes encoded in the genome revealed how much the gene repertoire of Ciona resembles those of vertebrates (e.g., Sasakura et al., 2003; Satou et al., 2003). Genome analyses also revealed genes that are specific to the ascidian (or tunicate) lineages. One of the unique genes encodes a protein that shows a high similarity to cellulose synthases of plants, fungi and bacteria (Nakashima et al., 2004). Ciona savignyi, a species related to
Ciona intestinalis, also possesses such a gene (Matthysse et al., 2004). The genes are named CesA, with the abbreviated species names such as $C i-C e s A$. Genes encoding the protein homologous to CesA were later detected in a non-ascidian tunicate, the larvaceans Oikopleula dioica (Sagane et al., 2010). The larvaceans do not settle, suggesting that they use cellulose for a purpose other than settlement. Larvaceans have a special tunic structure called a 'house,' which is used for collecting food efficiently. Cellulose fibers are present in the house (Kimura et al., 2001; Sagane et al., 2010).

The tunicate CesA proteins, which are seven-pass transmembrane proteins, have a unique domain composition (Fig. 2c). Their N-terminal region shows similarity to cellulose synthases of other organisms (Matthysse et al., 2004; Nakashima et al., 2004; Sagane et al., 2010). Tunicate CesA proteins have the signatures of the GT-2 cellulose synthase family. The C-terminal region of tunicate CesA, by contrast, shows similarity to the cellulase domains of the GH- 6 family. The coexistence of the domains whose deduced functions are completely opposite is a unique characteristic of tunicate cellulose synthases. However, the GH-6 cellulase domains of tunicate CesA have mutations in the residues that are essential for the catalysis of the reaction of GH-6, suggesting that the domains may not have the function of degrading cellulose (Matthysse et al., 2004).

The finding of CesA genes in the genomes of tunicates suggests that tunicates can produce cellulose for themselves. This hypothesis is supported by several lines of evidence. First, tunicate CesA genes are expressed in the epidermis (Matthysse et al., 2004; Nakashima et al., 2004; Sagane et al., 2010). The epidermis is the outermost tissue that covers the entire body, and it is in direct contact with the tunic; thus, the epidermis is the strongest candidate for the source of cellulose. Second, CesA gene of Ciona savignyi (Cs-CesA) can rescue the cellulose-absent phenotype of the bacterium Agrobacterium tumefaciens (Matthysse et al., 2004). Tunicates are therefore suspected to produce cellulose in the epidermis with CesA protein expressed in the tissue. Consistent with this idea, rosettes (the characteristic cellulose-producing complexes found in the plasma membrane of plant cells) were suggested to be present in the epidermis of the ascidian Metandrocarpa uedai (Kimura and Itoh, 1996).

\section{The functions of ascidian CesA and sessile life- style}

Although tunicate CesA proteins resemble those in other organisms, the similarity does not assure that the proteins function in cellulose synthesis in vivo. The roles of CesA in tunicates were shown in Ciona intestinalis by mutant analyses (Sasakura et al., 2005). CesA mutants exhibit a malformed tunic at the larval stage (Fig. 3). The malformation is caused 

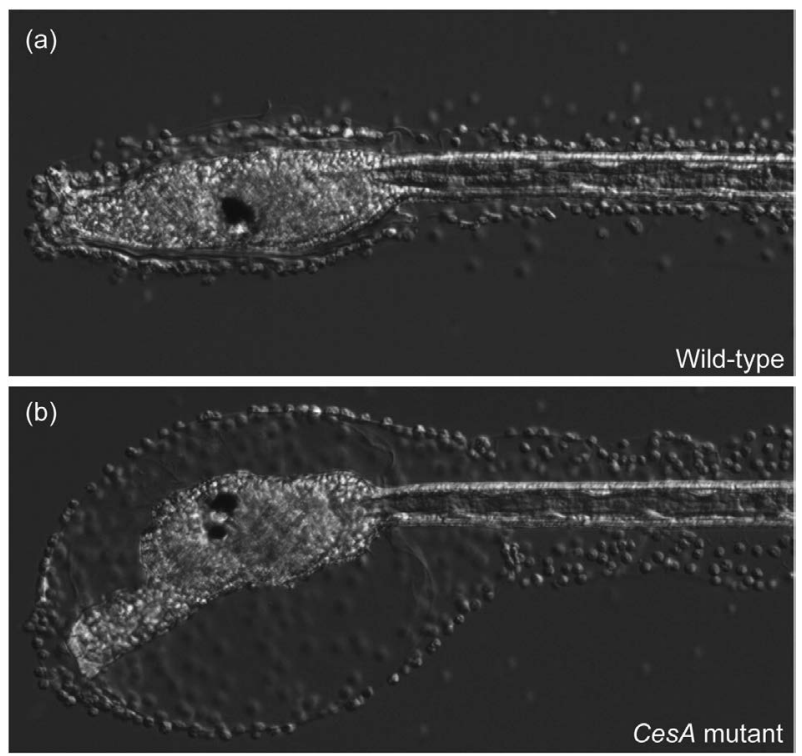

Fig. 3. The phenotypes of CesA mutant. a: A wild-type larva. b: A CesA mutant larva which does not have cellulose. Compared to the wild type, the tunic of the CesA mutant larva is swollen. The trunk of the mutant has a shape that is different from that of the wild type because of the untimely initiation of metamorphosis.

by the absence of cellulose fibers from the tunic, suggesting that Ciona intestinalis CesA (Ci-CesA) is indispensable for cellulose production in this ascidian, strengthening the role of $\mathrm{Ci}$-CesA as the cellulose synthase. After metamorphosis, the bodies of juvenile and adult CesA mutants are also covered by the tunic; however, the tunic is much softer than that of wildtype animals. Therefore, cellulose fiber is necessary to give the physical strength to the tunic and is also necessary for the appropriate shaping of the tunic. The functions of larvacean Ces $A$ were addressed in knockdown experiments, and the results demonstrated that $\operatorname{Ces} A$ is also necessary for the correct cellulose fiber formation in this tunicate (Sagane et al., 2010).

Observations of $C i$-Ces $A$ mutants indicated an unexpected role of cellulose in ascidians (Sasakura et al., 2005). CesA mutant larvae exhibit abnormal metamorphosis. Ascidian larvae do not start metamorphosis without settlement by the adhesive papillae. In this metamorphosis-prolonged situation, wild-type larvae do not change their morphology at all. CesA mutant larvae, during swimming, begin some metamorphic events that convert the morphology of their trunk (Fig. 3). Because CesA mutant larvae do not start tail regression if they do not adhere, their trunk looks like that of juveniles but they have a tail and continue swimming. The mutant was named swimming juvenile after the shape of the mutant larvae.

CesA mutant larvae have a decreased ability to adhere to a surface with their adhesive papillae. This is not because they do not have the mucus substrate at the papillae. Our observations suggest that they have the mucus substrate and they can start adhesion; however, they cannot maintain adhesion and are easily removed from the wall of culturing plate where they adhere. Therefore, although cellulose is not the mucus substance itself, cellulose is necessary for efficient adhesion.
CesA mutant larvae can somehow complete metamorphosis to become adults. The metamorphosed CesA mutants are viable until the reproductive stage, and their bodies showed no difference from that of the wild types except for the tunic.

Ces $A$ mutants are fertile because they can produce matured gametes. However, CesA mutant juveniles and adults have a weaker ability to adhere to substrates and they can be easily detached. The tunic of CesA mutants is jelly-like and can be removed with a gentle touch of our fingers. The celluloseabsent tunic is thus useless for defense against predators. The bodies of CesA mutants are covered with algae to an unusually high degree that is not observed in the wild types.

All of the phenotypes or abnormalities of CesA mutants have a strong relationship with the sessile lifestyle of ascidians. The weaker ability to adhere to a surface is observed in CesA mutants at both the larval and adult stages. Ascidian metamorphosis initiates sessile life through the loss of the locomotive organ (see Fig. 2a). Resisting the growth of organisms on the body is particularly necessary for sessile animals to keep their bodies clean. The firm tunic is the barrier for immotile ascidians to protect themselves from predators. The loss of cellulose results in defects in these aspects, all of which are required for initiating and continuing sessile life. Therefore, the acquisition of cellulose-synthesizing ability is suspected to have enabled the ancestor of ascidians (which is thought to be a free-swimming animal (Wada et al., 1998)) to evolve their unique sessile lifestyle. The success of this evolution may have enabled the ascidian group to diversify the most among the tunicate groups in the ocean.

By contrast, cellulose could be dispensable for the development, growth and reproduction of Ciona. The acquisition of cellulose was probably accidental, and therefore cellulose 
(a)

Streptomyces avermitilis genome

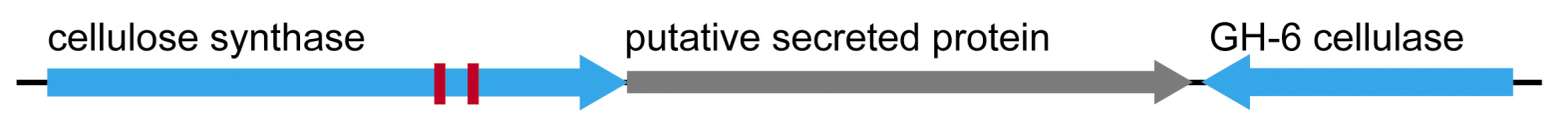

(b)
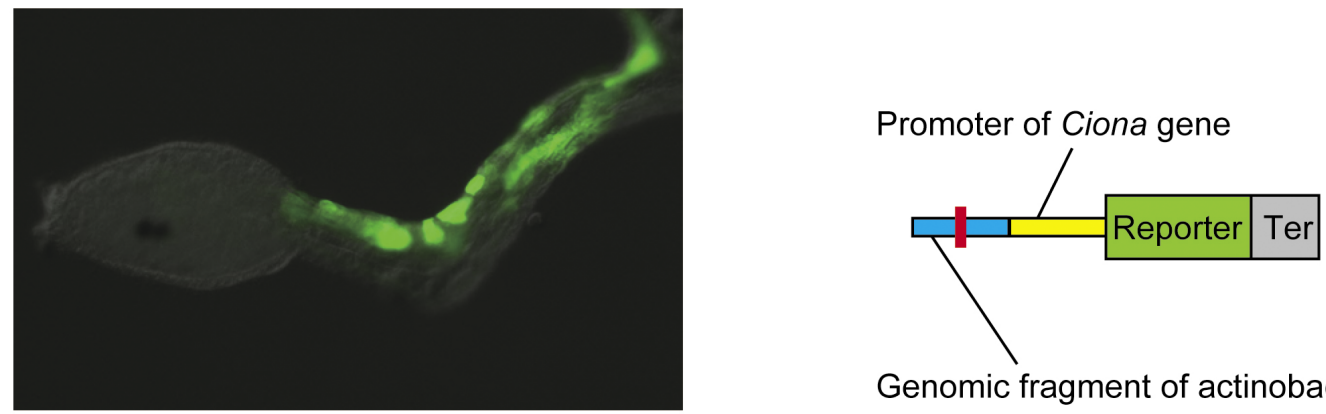

Genomic fragment of actinobacterium

(c)

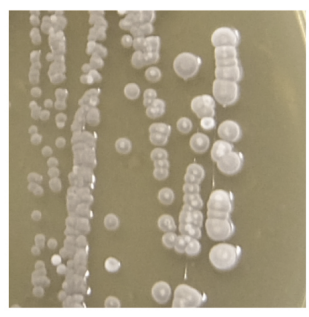

Actinobacteria

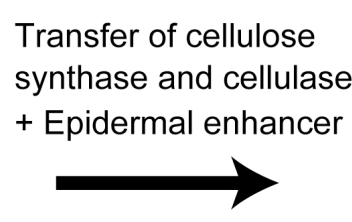

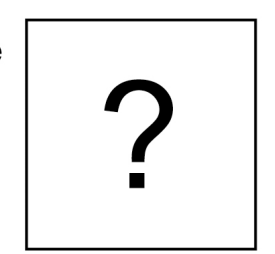

Ancestor of tunicates that possesses AP-2

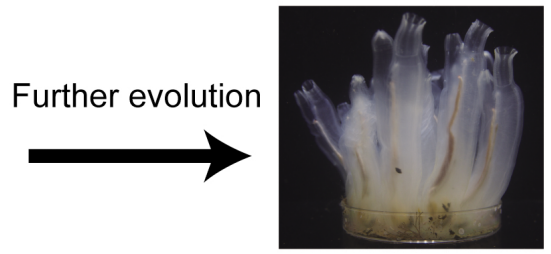

Extant ascidian

Fig. 4. Horizontal gene transfer of cellulose synthase gene in the tunicate lineage. a: The gene unit of cellulose synthase and cellulase genes in the genome of the actinobacterium Streptomyces avermitilis. Red bars: The position of the same sequence as the AP-2 binding site of Ciona CesA. Arrows: The direction of transcription. b: The actinobacterial genomic fragment containing the Ciona AP-2 binding site can act as the epidermal enhancer in Ciona in an AP-2 dependent manner. Green=expression of the reporter gene in the epidermis. The right panel is the schematic diagram of the DNA construct introduced in the Ciona larva shown in the left. Ter, transcription termination sequence. Red bar: The position of the same sequence as the AP-2 binding site of Ciona CesA. c: Schematic illustration of the horizontal gene transfer that occurred during the evolution of ascidians. Left panel: Photograph of the colonies of Streptomyces avermitilis provided by the Riken BioResource Center (resource no. 5070T).

could not penetrate into the core mechanisms responsible for the viability of tunicates. The viability of cellulose-absent ascidians is based on laboratory observations, and celluloseabsent ascidians may be less fit than wild types in the ocean because of their lower ability to settle.

\section{The origin of tunicate cellulose synthase}

Because animal groups related to tunicates do not possess the gene encoding CesA-like protein, it is natural to consider that $\operatorname{Ces} A$ gene was acquired specifically in the tunicate lineage after the group was separated from the vertebrate group (Fig. 1a). Horizontal gene transfer is the plausible mechanism of the sudden appearance of a new gene in the specific lineage. Like cellulose synthase, genes encoding GH-6 cellulase are not found in the animal lineage (Nakashima et al., 2004), suggesting that the ancestor of tunicates did not have the gene encoding GH-6 cellulase. GH-6 cellulase genes are found in the bacterial and eukaryotic (fungal) genomes. The independent transfer of cellulose synthase and GH- 6 cellulase genes in close proximity in the genome of the tunicates' ancestor is unlikely because of the low probability of such transfer events. It is thus suspected that both cellulose synthase and the cellulase domains of tunicate CesA were simultaneously acquired by a single horizontal gene transfer event from a bacterium or fungus.

Phylogenetic analyses suggest that tunicate CesA exhibits affinity to bacterial CesA and GH-6 cellulase proteins (Nakashima et al., 2004; Sagane et al., 2010). Some actinobacterial species encode both cellulose synthase and GH-6 cellulase at very close locations in the genome (Fig. 4a). Gene clusters of cellulose synthase and cellulase genes are common in bacteria; however, the pairing of cellulose synthase and GH-6 cellulase is unusual among bacteria (GH-8-type cellulase is usually a component of the gene units including cellulose syn- 
thase; Matthysse et al., 2004; Nakashima et al., 2004). Therefore, the gene unit of cellulose synthase and GH-6 cellulase seen in the actinobacterial genomes is a strong candidate as the origin of tunicate CesA.

Actinobacteria are a common bacterial group that live both in the ocean and on land. There is a report of an actinobacterium from the digestive tube of an ascidian (Bjerga et al., 2014). The ancestor of tunicates could thus have had the chance to acquire actinobacterial genes by contact with actinobacteria. The ubiquity of the bacterial group may be essential for the success of the horizontal gene transfer event whose probability is very low.

\section{The acquisition of the tissue-specific expression of CesA}

The critical step of the horizontal gene transfer is the acquisition of the expression of the acquired gene in the host organisms. Since genes can be functional after transcription in the appropriate spatiotemporal manner, foreign genes that have penetrated into a host genome must be expressed before gaining a function. This transcriptional activation is particularly essential for the gene transfers between phylogenetically distant organisms: because the regulative mechanisms of gene expression differ among organisms, genes given from a phylogenetically distant organism demand multiple modifications in their cis elements to be expressed. Tunicate $\operatorname{Ces} A$ is expressed in the epidermis (Matthysse et al., 2004; Nakashima et al., 2004; Sagane et al., 2010). In this case, the question is how tunicate $C e s A$ evolved to be expressed specifically in the epidermis.

To address this question, our group analyzed the enhancer of Ciona intestinalis CesA. The epidermal enhancer of $C i$ $\operatorname{Ces} A$ is simple: it possesses the single binding site of the transcription factor AP-2 (Imai et al., 2017; Ogura and Sasakura 2016) that is necessary for the epidermal expression of $C i-C e s A$ (Sasakura et al., 2016). This simplicity is unusual regarding tissue-specific expression during embryogenesis; usually enhancers have multiple binding sites of transcription factors to achieve tissue-specific expressions even in the relatively short cis elements of Ciona genes (Corbo et al., 1997; Kusakabe et al., 2004; Irvine et al., 2011).

The next question is how CesA acquired the AP-2 binding site at its upstream region. AP-2 is a conserved transcription factor among chordates, and it is responsible for the specification of ectoderm, which includes the epidermis (Eckert et al., 2005). The conservation of AP-2 suggests that the ancestor of tunicates already possessed AP-2 expressed in the epidermis. The binding site of AP-2 is known to be rich in G and C. Particularly, the AP-2 binding site of Ciona CesA is $5^{\prime}$-gectgcgggc-3' (Sasakura et al., 2016). Moreover, an approximately 100-base pair DNA stretch that flanks the AP-2 binding site of
CesA is also rich in GC. Because the genomes of tunicates are usually rich in $\mathrm{A}$ and $\mathrm{T}$ (Dehal et al., 2002), the de novo appearance of such a GC-rich element is unlikely.

The genomes of actinobacteria are usually rich in $\mathrm{G}$ and $\mathrm{C}$ (approx. 70\% GC; Bentley et al., 2002). This suggests that if tunicate CesA originated from the bacterial group, the gene was embedded in the GC-rich DNA stretch. The GC-rich DNA has a high probability of having a sequence similar to the AP-2 binding site in the proximal region of its gene body, although AP-2 is not encoded in the bacterial genome. Indeed, the gene units of cellulose synthase and GH-6 cellulase of some actinobacterial species have a sequence that is identical to the AP-2 binding site of Ciona CesA (Fig. 4a; Sasakura et al., 2016). The genome fragments of actinobacteria that include the identical sequence to the CesA AP-2 binding site(s) exhibit the epidermal enhancer activity in Ciona (Fig. 4b), suggesting that the actinobacterial genome had the potential to be the epidermal enhancer in the ascidian genome. Therefore, the likelihood of the success of the horizontal gene transfer of $\operatorname{Ces} A$ from actinobacteria to the ancestor of tunicates would be increased because of the simultaneous transfer of the gene body and the enhancer (Fig. 4c).

Moreover, a key factor of this success is that the enhancer provided by actinobacteria was likely to be an epidermal enhancer due to the curious mutuality between the GC-rich genome feature of the bacterial group and the presence of the epidermal transcription factor AP-2 that recognizes the GCrich element in the tunicates' ancestor. The epidermis is the tissue where cellulose can be used most efficiently compared to other internal organs, as is seen in the usage of this molecule in various organisms including plants, fungi and bacteria as the major component of the cell wall. For the protection of the bodies of organisms, the outermost tissue is the best region to be covered with a firm shield.

It must be stressed that the above information suggests one potential mechanism of the horizontal gene transfer of tunicate $\operatorname{Ces} A$ gene, and that this would not be the only plausible mechanism. Because the gene transfer could succeed just by chance, another explanation would be that the ancestor of tunicates was tremendously "lucky" to gain functional CesA. However, the fact that no other animal group can produce cellulose (even though the cellulose molecule is the most common organic molecule on Earth) suggests that attainment of the cellulose synthesis ability by horizontal gene transfer is difficult, but the presence of factor(s) that could increase the chance of the successful horizontal gene transfer could explain why the tunicate's ancestor attained this ability with evolution.

\section{Future perspectives}

The acquisition of cellulose synthesis ability could have 
served as the driving force for the ascidians' ancestor to evolve to a sessile lifestyle. Ascidians protect their soft body with a cellulose-containing membrane cover, and they can avoid being preyed upon even without moving. When cellulose is absent from the ascidian Ciona, the abnormal animals are unable to continue settlement even in mild conditions in the laboratory. These lines of evidence suggest that the sessile life of ascidians has been established based on cellulose.

Although there are several hypotheses regarding how cellulose synthesis ability came into the tunicate lineage, there are many issues that must be solved to completely understand the sessile lifestyle of ascidians. These issues include the following:

(1) Identification of the substance used in the mechanism of settlement. The first event in the settlement of ascidians is the adhesion by the adhesive papilla. The adhesive papilla has a mucus substance. The elucidation of this substance is necessary to understand larval adhesion. The characterization of the expression profile of the genes responsible for the production of the mucus will help determine whether the adhesive mechanism at the larval stage is the same as, or different from, that at the adult stage. If the adhesive mechanisms differ, the characterization of the adult adhesive substance is also required. This characterization will enable us to compare adhesive mechanisms among sessile animals to understand the conservation of animal sessility. The adhesive molecule(s) could also be an important target for the control of fouling by ascidians.

(2) The mechanisms of metamorphosis. Because metamorphosis is the necessary event for ascidians to start a sessile lifestyle, understanding the underlying mechanisms of metamorphosis is particularly important for elucidating how ascidians evolved the sessile lifestyle (Sasakura and Hozumi, 2018). Several studies have demonstrated the importance of neuronal networks for the initiation of metamorphosis (Coniglio et al., 1998; Kimura et al., 2003; Kamiya et al., 2014; Hirai et al., 2017), and the characterization of the neurons responsible for metamorphosis is necessary for a complete understanding of this process.

(3) Characterization of the pathways of cellulose production. The acquisition and expression of CesA is not the only step for animals to acquire the ability to produce cellulose, because organisms use multiple steps for synthesizing cellulose (Schneider et al., 2016). Because cellulose production starts in the epidermis at around the tailbud stage (Matthysse et al., 2004; Nakashima et al., 2004), the genes expressed in the epidermis at around this stage are strong candidates that encode proteins responsible for the production of cellulose with CesA.
(4) Conservation of the CesA expression mechanism among tunicates. Ciona CesA is expressed in the epidermis in an AP-2-dependent manner (Sasakura et al., 2016). If the CesA expression mechanism was passed on from the ancestor, the mechanism must be retained within all tunicates. The characterization of the expression mechanisms of CesA in the larvaceans and thaliaceans is required for a comprehensive understanding of the evolution of $\operatorname{Ces} A$ gene in tunicates.

(5) The identification of the origin of tunicate CesA. Although actinobacteria is the strongest candidate as the source of tunicate CesA, this has not been established because it is very difficult to estimate the lateral transfer of a gene that occurred in at least several hundred million years ago. It is not yet clear how this issue can be addressed, but answering the question of how the lateral gene transfer occurred is particularly important for understanding the evolution of the sessile lifestyle of ascidians.

\section{Acknowledgement}

I thank Drs. Shigeki Fujiwara, Yutaka Satou, Manabu Yoshida, and all members of the Maizuru Fishery Research Station of Kyoto University, Misaki Marine Biological Station, and the University of Tokyo for the collection of Ciona adults. This study was supported by Grants-in-Aid for Scientific Research from the Japan Society for the Promotion of Science (JSPS) and the Ministry of Education, Culture, Sports, Science and Technology (MEXT) to Y.S. This study was further supported by grants from the National Bioresource Project.

\section{Literature Cited}

Bentley, S. D., K. F. Chater, A.-M. Cerdeño-Tárraga, G. L. Challis, N. R. Thomson, K. D. James, D. E. Harris, M. A. Quail, H. Kieser, D. Harper, A. Bateman, S. Brown, G. Chandra, C. W. Chen, M. Collins, A. Cronin, A. Fraser, A. Goble, J. Hidalgo, T. Hornsby, S. Howarth, C.-H. Huang, T. Kieser, L. Larke, L. Murphy, K. Oliver, S. O’Neil, E. Rabbinowitsch, M.-A. Rajandream, K. Rutherford, S. Rutter, K. Seeger, D. Saunders, S. Sharp, R. Squares, S. Squares, K. Taylor, T. Warren, A. Wietzorrek, J. Woodward, B. G. Barrell, J. Parkhill and D. A. Hopwood (2002). Complete genome sequence of the model actinomycete Streptomyces coelicolor A2(2). Nature, 417, 141-147.

Bjerga, G. E. K., E. Hjerde, C. De Santi, A. K. Williamson, A. O. Smalas, N. P. Willassen and B. Altermark (2014). High quality draft genome sequence of Streptomyces sp. strain AW19M42 isolated from a sea squirt in Northern Norway. Stand. Genomic Sci., 9, 676-686.

Chambon, J. P., J. Soule, P. Pomies, P. Fort, A. Sahuquet, D. Alexandre, P. H. Mangeat and S. Baghdiguian (2002). Tail regression in Ciona intestinalis (Protochordate) involved a Caspase-dependent apoptosis event associated with ERK activation. Development, 129, 3105-3114.

Chambon, J. P., A. Nakayama, K. Takamura, A. McDougall and N. Satoh (2007). ERK- and JNK-signalling regulate gene networks that stimulate metamorphosis and apoptosis in tail tissue of ascidian tadpoles. Development, 134, 1203-1219. 
Cloney, R. A. (1982). Ascidian larvae and the events of metamorphosis. Amer. Zool., 22, 817-826.

Coniglio, L., A. Morale, C. Angelini and C. Falugi (1998). Cholinergic activation of settlement in Ciona intestinalis metamorphosing larvae. J. Exp. Zool., 280, 314-320.

Corbo, J. C., M. Levine and R. W. Zeller (1997). Characterization of a notochord-specific enhancer from the Brachyury promoter region of the ascidian, Ciona intestinalis. Development, 124, 589-602.

Dehal, P., Y. Satou, R. K. Campbell, J. Chapman, B. Degnan, A. De Tomaso, B. Davidson, A. Di Gregorio, M. Gelpke, D. M. Goodstein, N. Harafuji, K. E. Hastings, I. Ho, K. Hotta, W. Huang, T. Kawashima, P. Lemaire, D. Martinez, I. A. Meinertzhagen, S. Necula, M. Nonaka, N. Putnam, S. Rash, H. Saiga, M. Satake, A. Terry, L. Yamada, H. G. Wang, S. Awazu, K. Azumi, J. Boore, M. Branno, S. Chin-Bow, R. DeSantis, S. Doyle, P. Francino, D. N. Keys, S. Haga, H. Hayashi, K. Hino, K. S. Imai, K. Inaba, S. Kano, K. Kobayashi, M. Kobayashi, B. I. Lee, K. W. Makabe, C. Manohar, G. Matassi, M. Medina, Y. Mochizuki, S. Mount, T. Morishita, S. Miura, A. Nakayama, S. Nishizaka, H. Nomoto, F. Ohta, K. Oishi, I. Rigoutsos, M. Sano, A. Sasaki, Y. Sasakura, E. Shoguchi, T. Shin-i, A. Spagnuolo, D. Stainier, M. M. Suzuki, O. Tassy, N. Takatori, M. Tokuoka, K. Yagi, F. Yoshizaki, S. Wada, C. Zhang, P. D. Hyatt, F. Larimer, C. Detter, N. Doggett, T. Glavina, T. Hawkins, P. Richardson, S. Lucas, Y. Kohara, M. Levine, N. Satoh and D. S. Rokhsar (2002). The draft genome of Ciona intestinalis: Insight into chordate and vertebrate origins. Science, 298, 2157-2167.

Delsuc, F., H. Brinkmann, D. Chourrout and H. Philippe (2006). Tunicates and not cephalochordates are the closest living relatives of vertebrates. Nature, 439, 965-968.

Dufour, H. D., Z. Chettouh, C. Deyts, R. de Rosa, C. Goridis, J. S. Joly and J. F. Brunet (2006). Precraniate origin of cranial motoneurons. Proc. Natl. Acad. Sci. USA, 103, 8727-8732.

Eckert, D., S. Buhl, S. Weber, R. Jäger and H. Schorle (2005). The AP-2 family of transcription factors. Genome Biol., 6, 246.

Hirai, S., K. Hotta, Y. Kubo, A. Nishino, S. Okabe, Y. Okamura and H. Okado (2017). AMPA glutamate receptors are required for sensory-organ formation and morphogenesis in the basal chordate. Proc. Natl. Acad. Sci. USA, 114, 3939-3944.

Hirano, T. and H., Nishida (1997). Developmental fates of larval tissues after metamorphosis in ascidian Halocynthia roretzi. I. Origin of mesodermal tissues of the juvenile. Dev. Biol., 192, 199-210.

Hirano, T. and H., Nishida (2000). Developmental fates of larval tissues after metamorphosis in the ascidian, Halocynthia roretzi. II. Origin of endodermal tissues of the juvenile. Dev. Genes Evol., 210, 55-63.

Hirose, E., S. Kimura, T. Itoh and J. Nishikawa (1999). Tunic morphology and cellulosic components of Pyrosomas, Doliolids, and Salps (Thaliacea, Urochordata). Biol. Bull., 196, 113-120.

Horie, T., T. Kusakabe and M. Tsuda (2008). Glutamatergic networks in the Ciona intestinalis larva. J. Comp. Neurol., 508, 249-263.

Horie, T., M. Nakagawa, Y. Sasakura, T. G. Kusakabe and M. Tsuda (2010). Simple motor system of the ascidian larva: Neuronal complex comprising putative cholinergic and GABAergic/glycinergic neurons. Zoolog. Sci., 27, 181-190.

Ikuta, T. and H., Saiga (2007). Dynamic change in the expression of developmental genes in the ascidian central nervous system: Revisit to the tripartite model and the origin of the midbrainhindbrain boundary region. Dev. Biol., 312, 631-643.

Imai, K., H. Hikawa, K. Kobayashi and Y. Satou (2017). Tfap2 and Sox 1/2/3 cooperatively specify ectodermal fates in ascidian embryos. Development, 144, 33-37.

Irvine, S., D. Vierra, B. Millette, M. Blanchette and R. Holbert (2011). Expression of the Distalless-B gene in Ciona is regulated by a pan-ectodermal enhancer module. Dev. Biol., 353,
432-439.

Kamiya, C., N. Ohta, Y. Ogura, K. Yoshida, T. Horie, T. G. Kusakabe, H. Satake and Y. Sasakura (2014). Nonreproductive role of gonadotropin-releasing hormone in the control of ascidian metamorphosis. Dev. Dyn., 243, 1524-1535.

Kawai, N., Y. Ogura, T. Ikuta, H. Saiga, M. Hamada, T. Sakuma, T. Yamamoto, N. Satoh and Y. Sasakura (2015). Hox10-regulated endodermal strand cell migration is essential for development of the ascidian intestine. Dev. Biol., 403, 43-56.

Kimura, S. and T., Itoh (1996). New cellulose synthesizing complexes (terminal complexes) involved in animal cellulose biosynthesis in the tunicate Metandrocarpa uedai. Protoplasma., 194, 151163.

Kimura, S., C. Ohshima, E. Hirose, J. Nishikawa and T. Itoh (2001). Cellulose in the house of the appendicularian Oikopleura rufescens. Protoplasma, 216, 71-74.

Kimura, Y., M. Yoshida and M. Morisawa (2003). Interaction between noradrenaline or adrenaline and the $\beta_{1}$-adrenergic receptor in the nervous system triggers early metamorphosis of larvae in the ascidian Ciona savignyi. Dev. Biol., 258, 129-140.

Kusakabe, T., R. Yoshida, Y. Ikeda and M. Tsuda (2004). Computational discovery of DNA motifs associated with cell typespecific gene expression in Ciona. Dev. Biol., 276, 563-580.

Matthysse, A. G., K. Deschet, M. Williams, M. Marry, A. R. White and W. C. Smith (2004). A functional cellulose synthase from ascidian epidermis. Proc. Natl. Acad. Sci. USA, 101, 986-991.

Nakashima, K., L. Yamada, Y. Satou, J. Azuma and N. Satoh (2004). The evolutionary origin of animal cellulose synthase. Dev. Genes Evol., 214, 81-88.

Nakazawa, K., T. Yamazawa, Y. Moriyama, Y. Ogura, N. Kawai, Y. Sasakura and H. Saiga (2013). Formation of the digestive tract in Ciona intestinalis includes two distinct morphogenic processes between its anterior and posterior parts. Dev. Dyn., 242, $1172-1183$.

Nicol, D. and I. A., Meinertzhagen (1988). Development of the central nervous system of the larva of the ascidian, Ciona intestinalis L. II. Neural plate morphogenesis and cell lineages during neurulation. Dev. Biol., 130, 737-766.

Nishino, A., S. A. Baba and Y. Okamura (2011). A mechanism for graded motor control encoded in the channel properties of the muscle ACh receptor. Proc. Natl. Acad. Sci. USA, 108, 25992604.

Ogura, Y. and Y., Sasakura (2016). Developmental control of cellcycle compensation provides a switch for patterned mitosis at the onset of chordate neurulation. Dev. Cell, 37, 148-161.

Ogura, Y., A. Sakaue-Sawano, M. Nakagawa, N. Satoh, A. Miyawaki and Y. Sasakura (2011). Coordination of mitosis and morphogenesis: Role of a prolonged G2 phase during chordate neurulation. Development, 138, 577-587.

Ranby, B. G. (1952). Physico-chemical investinations on animal cellulose (Tunicin). Ark. Kemi., 4, 241-248.

Ryan, K., Z. Lu and I. Meinertzhagen (2016). The CNS connectome of a tadpole larva of Ciona intestinalis (L.) highlights sidedness in the brain of a chordate sibling. Elife, 5, e16962.

Sagane, Y., K. Zech, J. M. Bouquet, M. Schmid, U. Bal and E. M. Thompson (2010). Functional specialization of cellulose synthase genes of prokaryotic origin in chordate larvaceans. Development, 137, 1483-1492.

Sasakura, Y. and A., Hozumi (2018). Formation of adult organs through metamorphosis in ascidians. Wiley Interdiscip. Rev. Dev. Biol., 7, e304.

Sasakura, Y., L. Yamada, N. Takatori, Y. Satou and N. Satoh (2003). A genomewide survey of developmentally relevant genes in Ciona intestinalis. VII. Molecules involved in the regulation of cell polarity and actin dynamics. Dev. Genes Evol., 213, 273-283.

Sasakura, Y., K. Nakashima, S. Awazu, T. Matsuoka, A. Nakayama, J. Azuma and N. Satoh (2005). Transposon-mediated insertional 
mutagenesis revealed the functions of animal cellulose synthase in the ascidian Ciona intestinalis. Proc. Natl. Acad. Sci. USA, 102, 15134-15139.

Sasakura, Y., Y. Ogura, N. Treen, R. Yokomori, S. J. Park, K. Nakai, H. Saiga, T. Sakuma, T. Yamamoto, S. Fujiwara and K. Yoshida (2016). Transcriptional regulation of a horizontally transferred gene from bacterium to chordate. Proc. Biol. Sci., 283, 20161712.

Satoh, N. (2003). The ascidian tadpole larva: Comparative molecular development and genomics. Nat. Rev. Genet., 4, 285-295.

Satoh, N., D. Rokhsar and T. Nishikawa (2014). Chordate evolution and the three-phulum system. Proc. Biol. Sci., 281, 20141729.

Satou, Y., K. Imai, M. Levine, Y. Kohara, D. Rokhsar and N. Satoh (2003). A genomewide survey of developmentally relevant genes in Ciona intestinalis. I. Genes for bHLH transcription factors. Dev. Genes Evol., 213, 213-221.

Schneider, R., T. Hanak, S. Persson and C. Voigt (2016). Cellulose and callose synthesis and organization in focus, what's new? Curr. Opin. Plant Biol., 34, 9-16.

Shirae-Kurabayashi, M., T. Nishikata, K. Takamura, K. J. Tanaka, C. Nakamoto and A. Nakamura (2006). Dynamic redistribution of vasa homolog and exclusion of somatic cell determinants during germ cell specification in Ciona intestinalis. Development, 133, 2683-2693.

Stolfi, A. and M., Levine (2011). Neuronal subtype specification in the spinal cord of a protovertebrate. Development, 138, 9951004.

Takamura, K. (1998). Nervous network in larvae of the ascidian Ciona intestinalis. Dev. Genes Evol., 208, 1-8.

Wada, H., H. Saiga, N. Satoh and P. W. H. Holland (1998). Tripartite organization of the ancestral chordate brain and the antiquity of placodes: Insights from ascidian Pax-2/5/8, Hox and Otx genes. Development, 125, 1113-1122. 